
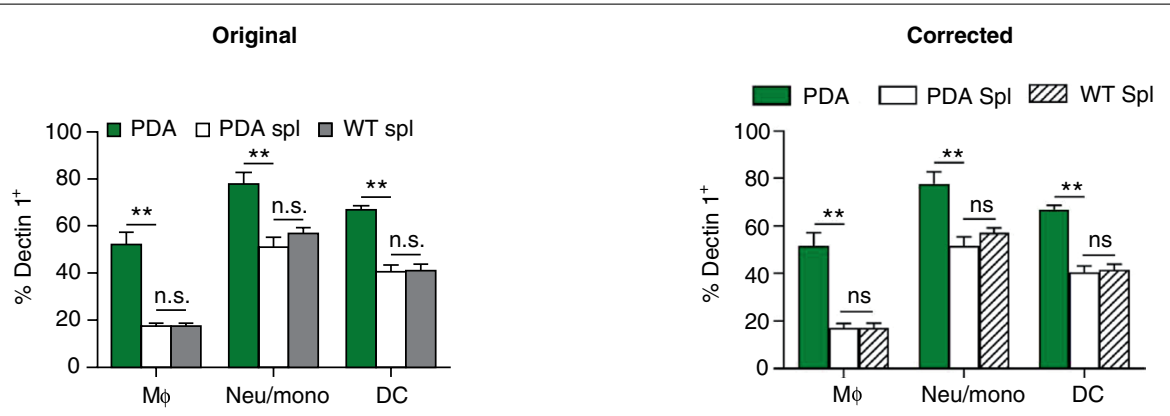

Fig. 1d | Original and Corrected.
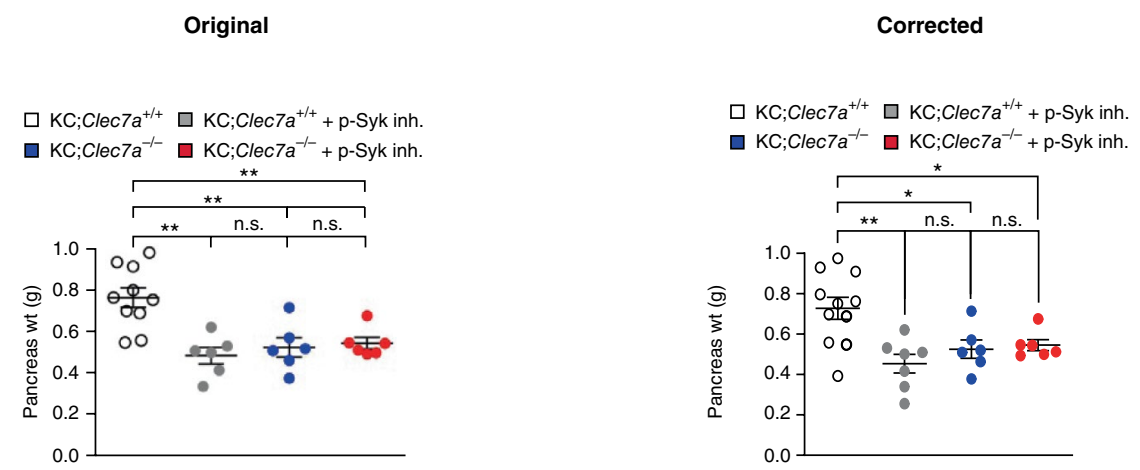

Fig. 2d | Original and Corrected.

Published online: 29 November 2021

https://doi.org/10.1038/s41591-021-01428-0

(๑) The Author(s), under exclusive licence to Springer Nature America, Inc. 2021

\title{
Publisher Correction: Clinical evolution, genetic landscape and trajectories of clonal hematopoiesis in SAMD9/SAMD9L syndromes
}

Sushree S. Sahoo, Victor B. Pastor, Charnise Goodings, Rebecca K. Voss, Emilia J. Kozyra @ , Amina Szvetnik, Peter Noellke D, Michael Dworzak, Jan Starý, Franco Locatelli, Riccardo Masetti, Markus Schmugge,

Barbara De Moerloose (D), Albert Catala, Krisztián Kállay, Dominik Turkiewicz, Henrik Hasle @ , Jochen Buechner (D),

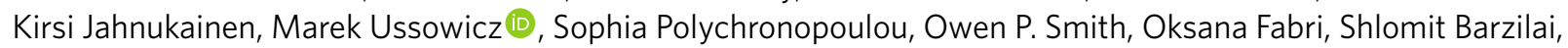
Valerie de Haas, Irith Baumann, Stephan Schwarz-Furlan, the European Working Group of MDS in Children

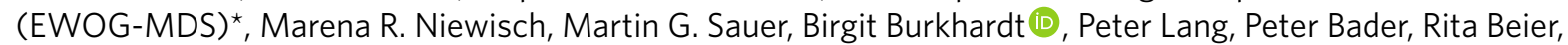
Ingo Müller (1), Michael H. Albert (10, Roland Meisel, Ansgar Schulz (D), Gunnar Cario, Pritam K. Panda (1), Julius Wehrle (1), Shinsuke Hirabayashi, Marta Derecka, Robert Durruthy-Durruthy, Gudrun Göhring, Ayami Yoshimi-Noellke, Manching Ku(D), Dirk Lebrecht, Miriam Erlacher (1), Christian Flotho, Brigitte Strahm (D), Charlotte M. Niemeyer (1) and Marcin W. Wlodarski (D)

Correction to: Nature Medicine https://doi.org/10.1038/s41591-021-01511-6, published online 7 October 2021.

In the version of this Article initially published, there were errors in Fig. 1. Specifically, in the top right box in Fig. 1a, the text now reading " $n=25$ " mistakenly read " 125 " in the original publication. Further, in the four graphs of Fig. 1e, the colored labels "Wild-type," "SAMD9/9L $L^{\text {mut" }}$ and "GATA2 $2^{\text {mut" }}$ were misaligned to the $n$ and $P$ values at their right. These errors have been corrected in the online version of the article.

${ }^{*}$ A list of members and their affiliations appears online.

Published online: 19 November 2021

https://doi.org/10.1038/s41591-021-01632-y

(c) The Author(s), under exclusive licence to Springer Nature America, Inc. 2021 